Supporting Information

\title{
NIR-Triggered Blasting Nanovesicles for Targeted Multimodal \\ Image-Guided Synergistic Cancer Photothermal and \\ Chemotherapy
}

Zeyu Xiao§, Yuanyuan You§, Yiyong Liu§, Lizhen He, Dong Zhang, Qingqing Cheng, Dan Wang, Tianfeng Chen*, Changzheng Shi*, Liangping Luo*

Medical Imaging Center, The First Affiliated Hospital, and Department of Chemistry, Jinan University, Guangzhou 510632, P. R. China.

The Guangzhou Key Laboratory of Molecular and Functional Imaging for Clinical Translation, Jinan University, Guangzhou 510632, P. R. China.

$\S$ These authors contributed equally to this work.

${ }^{*}$ To whom correspondence should be addressed:

* Liangping Luo, Medical Imaging Center, The First Affiliated Hospital of Jinan University, Guangzhou 510632, P. R. China.; E-mail: tluolp@jnu.edu.cn

${ }^{*}$ Changzheng Shi, Medical Imaging Center, The First Affiliated Hospital of Jinan University, Guangzhou 510632, P. R. China.; E-mail: sczcn@126.com

${ }^{*}$ Tianfeng Chen, The First Affiliated Hospital, and Department of Chemistry, Jinan University, Guangzhou 510632, P. R. China.; E-mail: tchentf@jnu.edu.cn 


\section{Materials and Method}

Materials. 1-Ethyl-3-(3-dimethylaminopropyl) carbodiimide hydrochloride (EDC·HCl), dimethyl sulfoxide (DMSO), nystatin, NaN3, 2-deoxy-D-glucose (DOG), sucrose, dynasore, N-hydroxysuccinimide (NHS), dihydroethidium (DHE), MTT, and Nembutal were obtained from Sigma-Aldrich. Acetone, tetraethyl orthosilicate (TEOS), 3-aminopropyltriethoxysilane (APTES), PFH, iron(III) 2,4-pentanedionate, (PLGA), and anhydrous benzyl alcohol were purchased from Aladdin Chemistry Co., Ltd. Hexadecyl trimethyl ammonium bromide (CTAB) and cisplatin were obtained from Shanghai Macklin Biochemical Co., Ltd. RGD was purchased from GL Biochem (Shanghai) Co., Ltd. MitoTracker Green and LysoTracker were obtained from Life Technologies. Hematin/Prussian blue was obtained from Beijing Saichi Biotechnology Co., Ltd. Anti-Ki67 and TUNEL assay kits were purchased from Cell Signaling or Sigma.

Synthesis of $\mathrm{Fe}_{3} \mathrm{O}_{4}$. First, $123.6 \mathrm{mg}$ of iron(III) 2,4-pentanedionate was dissolved in $4.5 \mathrm{~mL}$ of anhydrous benzyl alcohol and heated to $60{ }^{\circ} \mathrm{C}$ for 5 minutes. The temperature was increased to $210{ }^{\circ} \mathrm{C}$ for 10 minutes, then cooled to $60{ }^{\circ} \mathrm{C}$, and this temperature was maintained for 10 minutes. After cooling to $40{ }^{\circ} \mathrm{C}$ under natural conditions, the mixture was washed with acetone 3 times. The $\mathrm{Fe}_{3} \mathrm{O}_{4}$ sample was dispersed in $10 \mathrm{~mL}$ of ethanol at $4{ }^{\circ} \mathrm{C}$ for storage.

Characterization of PFe-PFH@SiO 2 and PFe-PFH@SiO $-P A N I . \quad P F e-$ PFH@SiO2 and PFe-PFH@SiO2-PANI were characterized by transmission electron microscopy (TEM) (Philips, TEANAI-10), scanning electron microscopy (SEM) (Horiba, EX-250), Raman spectroscopy (Horiba, LabRAM HR Evolution), UV-Vis spectroscopy (UH-4150 Spectrophotometer, Hitachi), Fourier transform infrared (FTIR) spectroscopy (Bruker, Equinox 55 IR), X-ray photoelectron spectroscopy (XPS) (Thermo Scientific, ESCALAB 250Xi) and a Zetasizer particle size analyzer (Malvern, Nano Nano-ZS). Inductively coupled plasma mass spectrometry (ICP-MS) was used to determine the concentrations of iron and cisplatin. 
Cell Culture. The human lung carcinoma cell line A549 and normal human lung fibroblast cell line WI-38 were purchased from the American Type Culture Collection (ATCC, Manassas, VA) and cultured in DMEM (Gibco) supplemented with 10\% fetal bovine serum (FBS; Gibco) at $37^{\circ} \mathrm{C}$ in a $5 \% \mathrm{CO}_{2}$ incubator.

In vitro cellular uptake of PFe-PFH@SiO 2 PANI. Cellular uptake of the nanosystem was investigated by flow cytometry as we previously described. In brief, cells were seeded in 6-well plates with $24 \mathrm{~h}$ incubation. After that, medium was pipetted out and serum-free medium was replenished. Then, FITC-labeled PFe-PFH@SiOzPANI was added. After incubation, cells were collected and rinsed with PBS for the analysis of flow cytometry.

Intracellular localization of PFe-PFH@SiO2-PANI. The intracellular localization of PFe-PFH@SiO2-PANI in A549 cells was studied as previously described. ${ }^{43}$ In brief, A549 cells were seeded in $2 \mathrm{~cm}$ dishes with $24 \mathrm{~h}$ incubation. After that, PFe-PFH@SiO 2-PANI was added at specific time intervals (2, 4, 8 and 12 hours). LysoTracker Green and DAPI were added at the corresponding times before observation via fluorescence microscopy (Nikon Eclipse 80i, Japan).

Apoptosis analysis. For analysis of cell apoptosis, A549 cells were plated in 6 well plates with a density of $3 \times 10^{5}$ cells/well. After cell adherence, the cells treated with different drug for $24 \mathrm{~h}$ at $37{ }^{\circ} \mathrm{C}$. Then, after removing from the medium and washing with cold PBS, the cells were stained with Annexin V and PI according to the experimental method in the assay kit (Solarbio) specification. The cell apoptosis was analyzed using a Beckman CytoFLEX S flow cytometer.

Animals and tumor xenograft model. All work performed on animals was approved by the Committee on Use and Care of Animals at Jinan University. Male BALB/c nude mice (4 5 weeks old, body weight 18 20 g) were purchased from Beijing HFK Biology Technology Co. Ltd. and maintained in a specific pathogen-free environment. A549 cells $\left(2 \times 10^{6} \mathrm{~mL}^{-1}\right)$ in $100 \mu \mathrm{L}$ of serum-free medium were 
subcutaneously injected into the right hind leg of each mouse to establish a murine lung cancer model.

Biochemical and histological analysis. After three weeks of treatment, the mice were sacrificed, and blood samples were collected from the heart to measure biochemical indexes, including blood urea nitrogen (BUN), creatinine (CREA), alanine aminotransferase (ALT) and creatine kinase (CK), with a Hitachi 7600 biochemical analyzer. Additionally, the collected organs, including the heart, liver, spleen, lung, kidney and tumor, were fixed, embedded and sectioned followed by staining with hematoxylin and eosin (H\&E) for pathological observation under an optical microscope (Nikon, Japan). The detection of Ki67 and TUNEL staining was determined via immunofluorescence analysis as we previously described. 


\section{Experimental section}
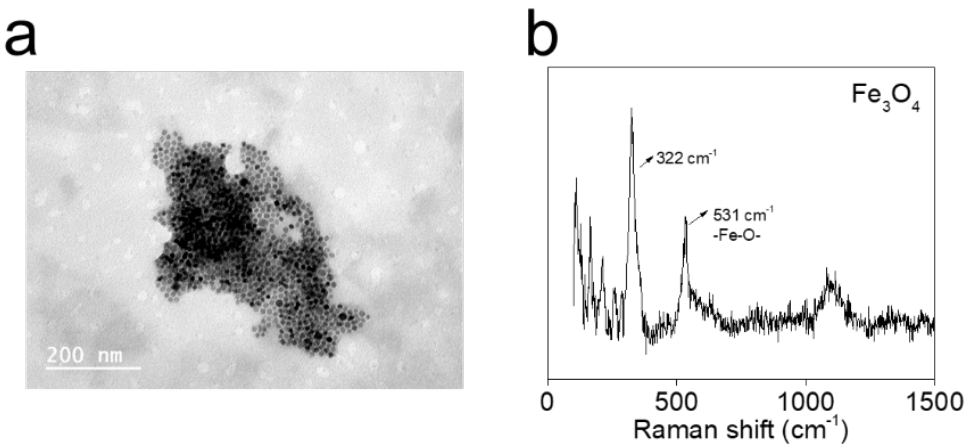

Figure S1. The transmission electronic microscopy(a) and Raman spectrum (b) of $\mathrm{Fe}_{3} \mathrm{O}_{4}$ nanoparticle. 


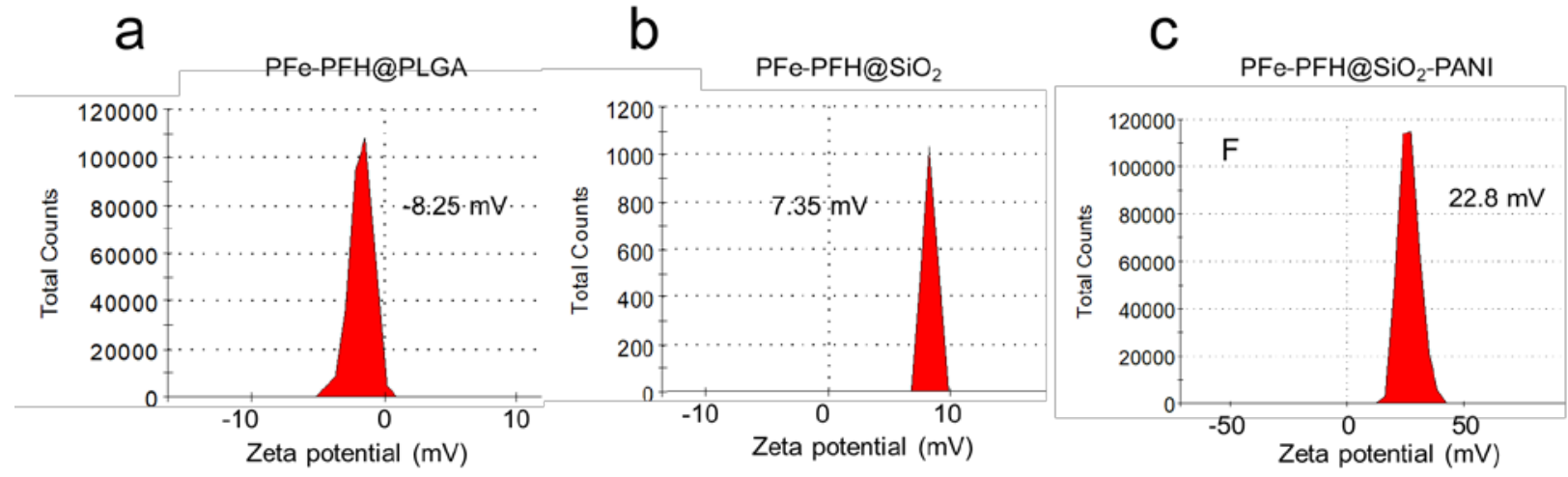

Figure S2. Zeta potential of PFe-PFH@PLGA (a), PFe-PFH@SiO2 (b) and PFePFH@SiO2-PANI (c). 
a

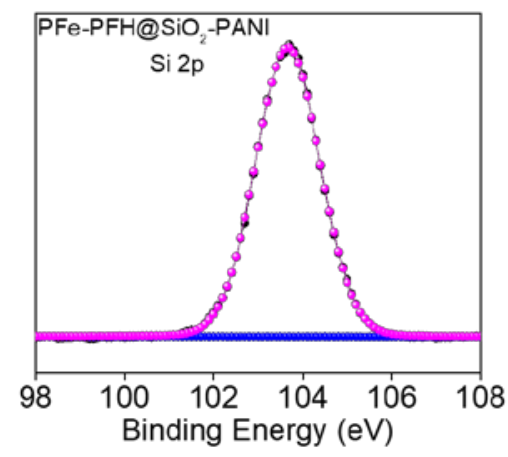

b

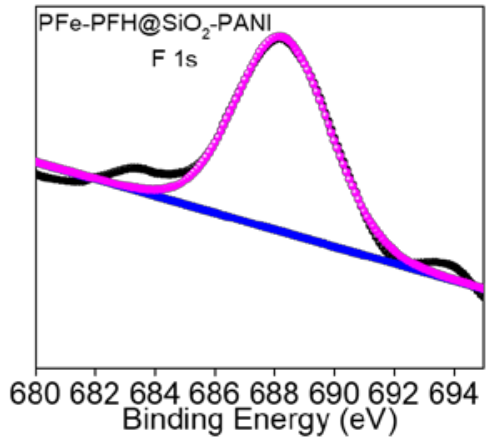

Figure S3. The high-resolution spectrum Si 2p (a) and F1s (b) of PFe-PFH@SiO2PANI. 

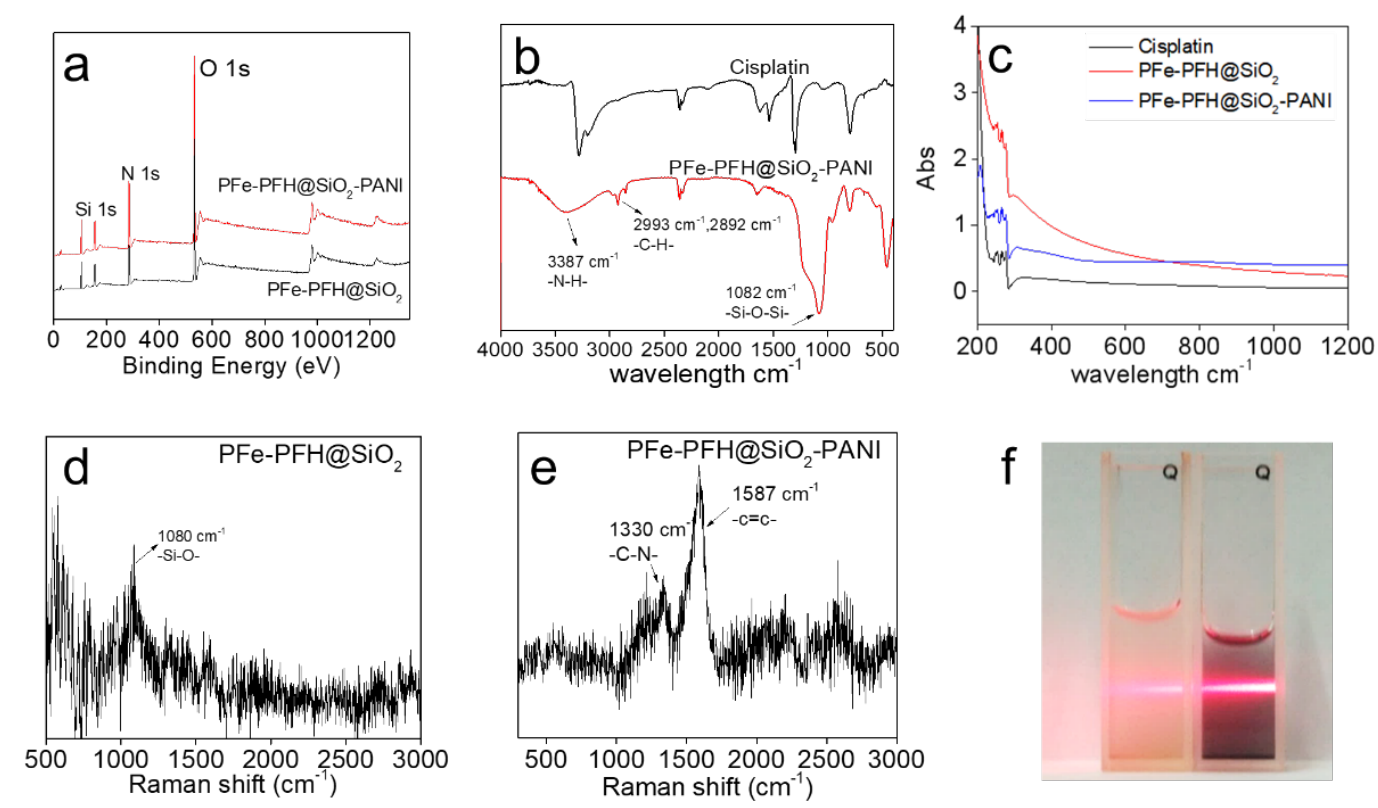

Figure S4. High-resolution XPS spectra showing the binding energies of PFePFH@SiO 2 and PFe-PFH@SiO 2 -PANI (a), the results of Raman spectroscopy and UVvis spectra of the PFe-PFH@SiOz-PANI nanosystem (b, c, d and e). The Tyndall effect is observed in the suspension of nanovesicles (f). 


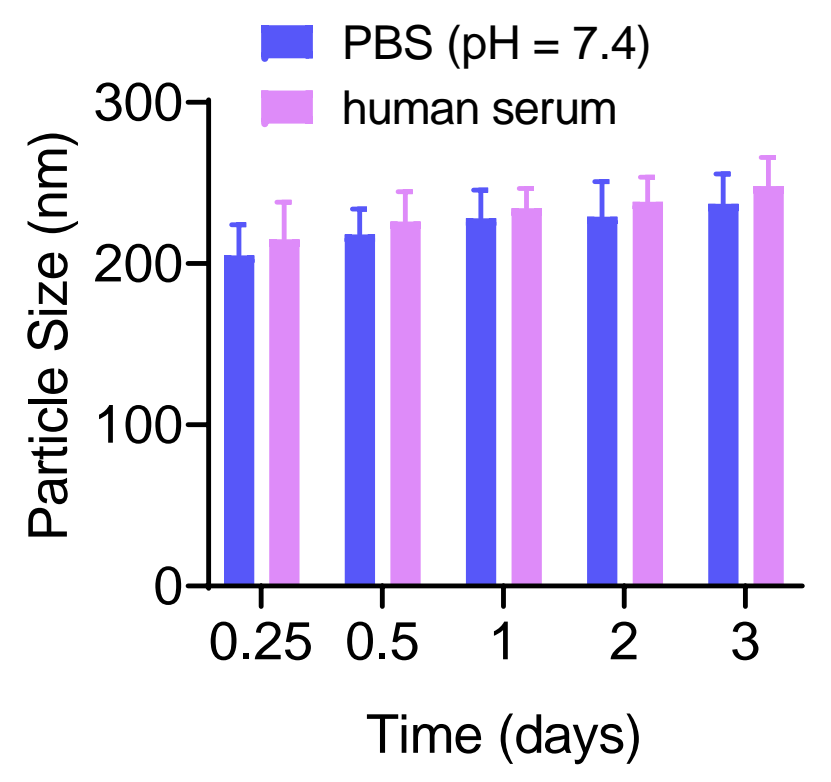

Figure S5. Particle sizes of PFe-PFH@SiO 2 -PANI after incubation in PBS and human serum at different time points. All data are shown as mean \pm S.D. $(n=3)$. 


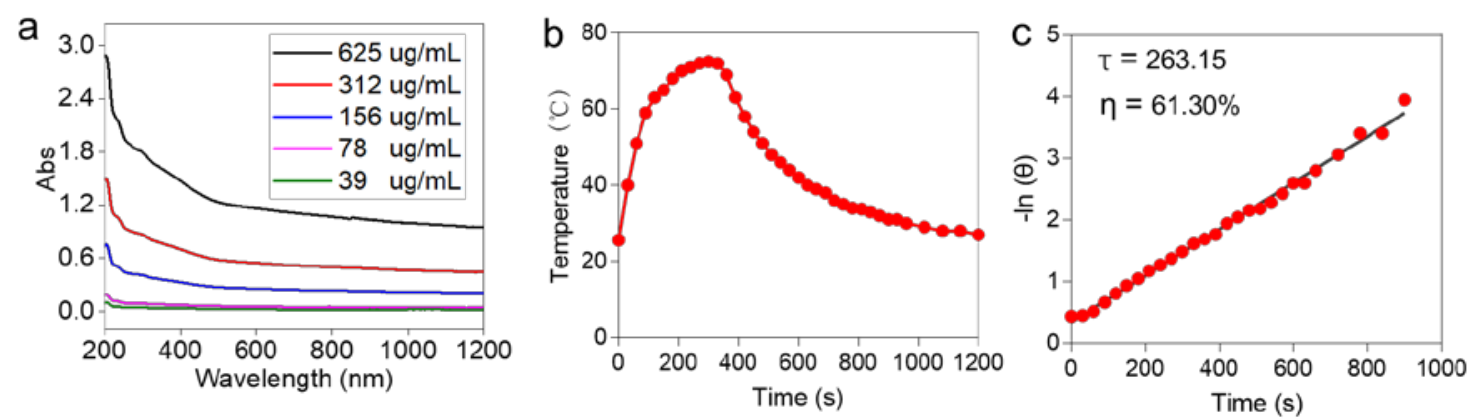

Figure S6. UV spectra of PFe-PFH@SiO2-PANI with different concentrations (a). Photothermal effect of PFe-PFH@SiO2-PANI nanosystem dispersion with a power density of $1 \mathrm{~W} / \mathrm{cm}^{2}$ at $808 \mathrm{~nm}$ and the laser was switched off after irradiation for $300 \mathrm{~s}$ (b). Thermal equilibrium time constant of the system is determined by applying the linear time data versus negative natural logarithm of the driving force temperature obtained from the cooling stage of (c). 


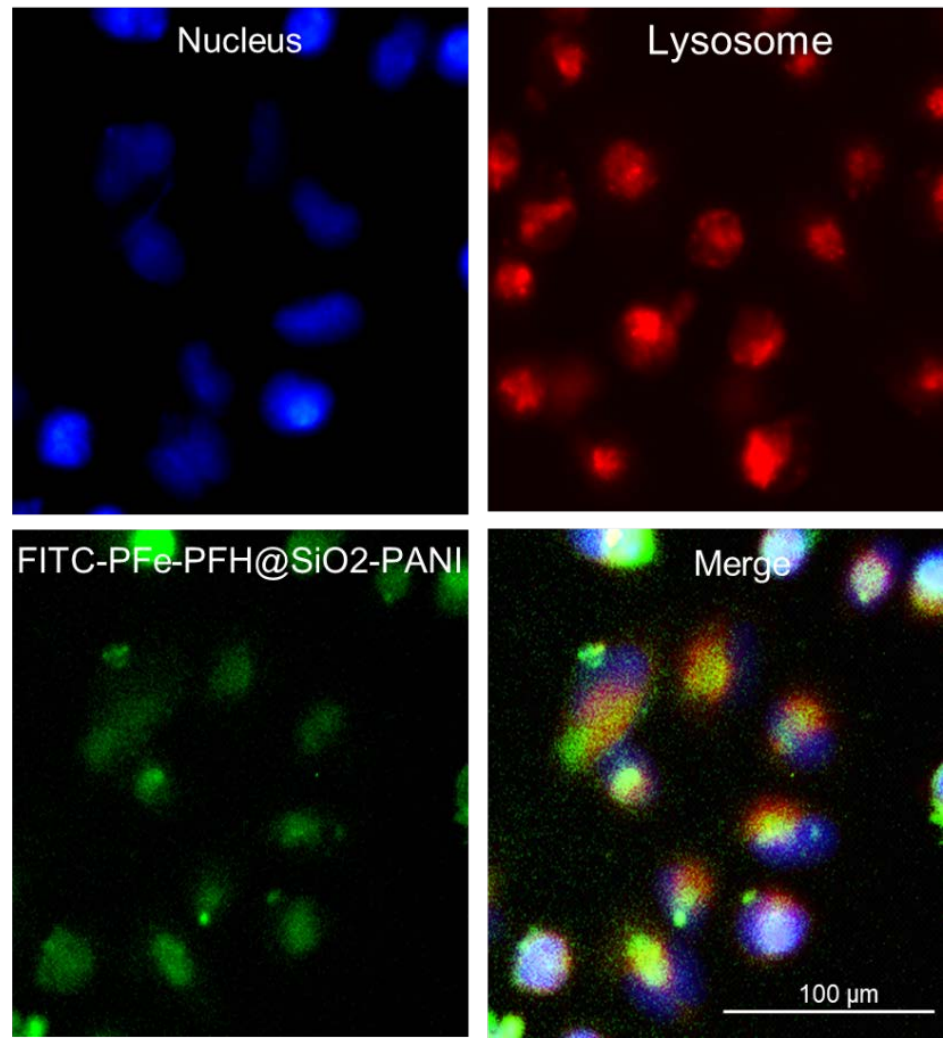

Figure S7. The localization of PFe-PFH@SiO2-PANI in A549 cells. 


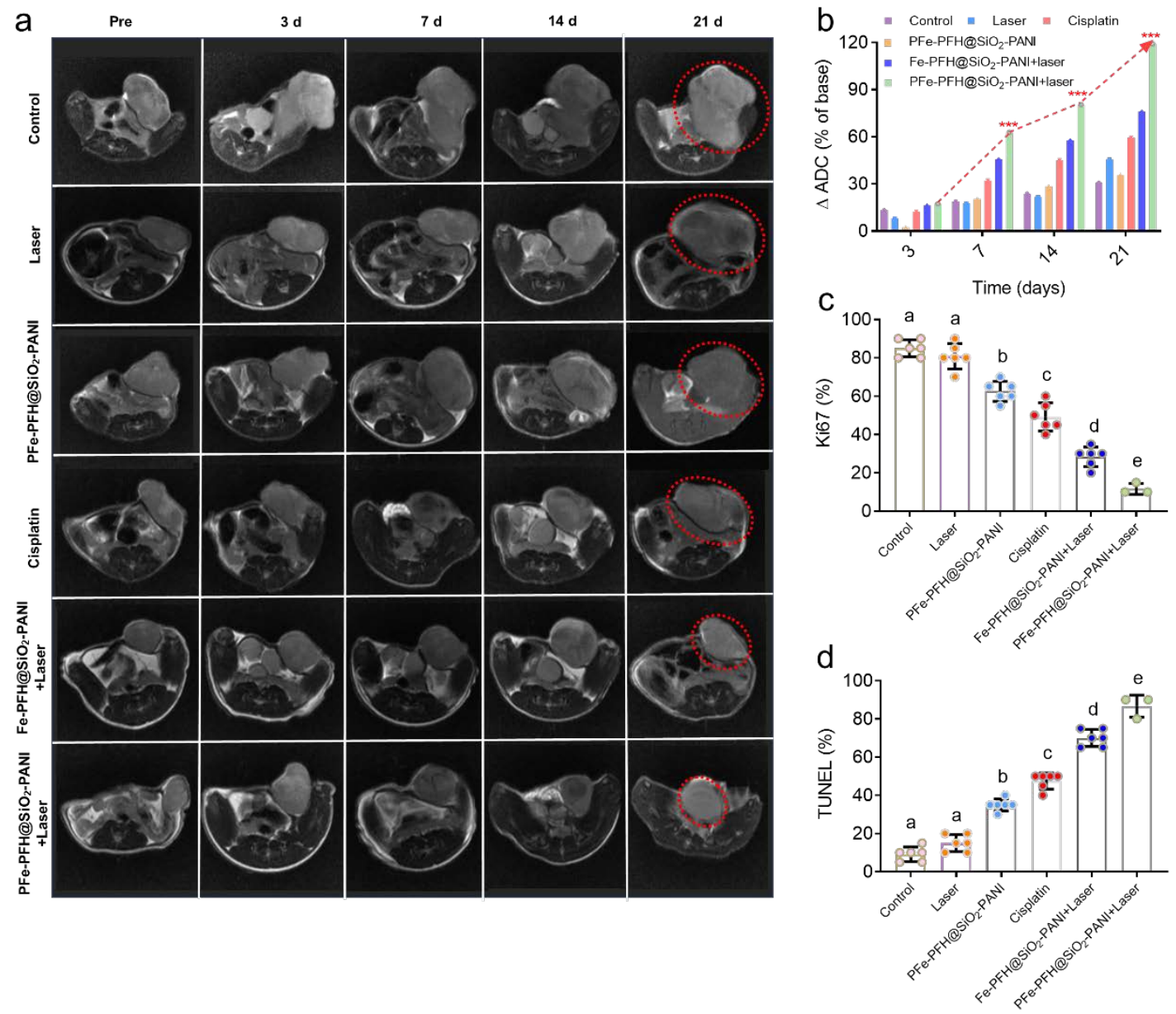

Figure S8. The $\mathrm{T}_{2}$-weighted MR imaging (a) and the ADC change (\%) of their baseline values (b) at 3 days, 7 days, 14 days and 21 days of the control group, laser group, cisplatin group, PFe-PFH@SiO2-PANI group, Fe-PFH@SiO2-PANI+Laser and the PFe-PFH@SiO2/PANI+Laser group. The quantitative analyses of ki67 and TUNEL staining are shown in (c) and (d). Bars with different characteristics are statistically different at the $P<0.05$ level. All data are shown as mean \pm S.D. $(n=5)$. 